\title{
Intestinal Schistosomiasis Caused by Schistosoma japonicum: A Literature Review
}

\author{
D.J. Rivadeneira' ${ }^{1 *}$, H.S. Luo' \\ 'Department of Gastroenterology, Renmin Hospital, Wuhan University, China
}

\section{Article Info}

\section{Article Notes}

Received: December 28, 2018

Accepted: February 1, 2019

\section{${ }^{*}$ Correspondence:}

D.J. Rivadeneira, Department of Gastroenterology, Renmin Hospital, Wuhan University, 430060 China;

Email: dany_ma13@hotmail.com

${ }^{-} 2019$ Rivadeneira DJ. This article is distributed under the terms of the Creative Commons Attribution 4.0 International License.

\section{Key words:}

Schistosoma japonicum

Intestinal schistosomiasis

Parasite egg

Endemic

Neglected tropical disease

\begin{abstract}
Intestinal schistosomiasis caused by the Schistosoma japonicum is located mainly in the East Asian region. Schistosomiasis is part of the neglected tropical diseases that affects mostly the poor population; although its incidence has dropped in these years, schistosomiasis caused by $S$. japonicum still is a prevalent disease. Adult worms reside in the mesenteric veins and excrete eggs that migrate through the intestinal wall and pass out with the stool. The clinical manifestations depend on the stage of the disease, the intestinal schistosomiasis mostly affects the colon, but it can also affect the small intestine. This review's purpose is to highlight the background and importance of the intestinal manifestations caused by Schistosoma japonicum.
\end{abstract}

Keywords: Schistosoma japonicum, Intestinal schistosomiasis, Parasite egg, Endemic, Neglected tropical disease

Introduction

Neglected tropical diseases (NTD) primarily affect the poorest and most vulnerable populations in the world, they are caused by infectious and parasitic agents ${ }^{1}$. Currently the NTD are affecting 41.4 billion people, causing approximately 35,000 deaths per day worldwide ${ }^{2}$. These "neglected" diseases are due to under-financing and low recognition by the pharmaceutical industries ${ }^{3}$.

Schistosomiasis is a NTD caused by infection with parasitic blood flukes. It is also known as "bilharziasis" after Theodor Bilharz, who first identified the parasite in $1852^{4}$. The WHO reported that 258 million people worldwide were infected with trematodes of the genus schistosoma, and required treatment and preventive regime ${ }^{5}$.

The distribution of Schistosoma japonicum (discovered in Japan) is mainly in China, the Philippines and Indonesia. China is endemic for $S$. japonicum with the oncomelania snail as an intermediate; the invasive snail Biomphalaria straminea is an intermediate host of $S$. mansoni that was first discovered in 1981 in Southern China ${ }^{6}$.

\section{Epidemiology}

Schistosomiasis is a prevalent infection in tropical and subtropical areas, especially in communities without adequate sanitation and safe drinking water ${ }^{7}$. In Asia the agricultural, fishing, and domestic activities in infested water are the main source of exposure; and introduction of this disease in urban areas is secondary to migration and the rise in eco-tourism ${ }^{8}$.

Schistosoma mansoni and S. haematobium are found in Africa and Middle East, S. japonicum and S. mekongi are found in the East 
Hemisphere, and S. intercalatum is found in parts of central Africa9. Schistosomiasis is considered the third most devastating tropical disease in Africa, South America, the Caribbean, Middle East and Asia ${ }^{10}$.

S. japonicum has been found along the Yangtze River Basin in China, the southern and eastern islands of the Philippines, and in central Sulawesi, Indonesia. According to a Chinese report for the World Health Organization, "S. japonicum infected 45 species of animals and remained endemic in seven provinces" ${ }^{\prime 1}$ and after decades of intensive control this disease still remains a public health problem. China is among the target countries to eliminate schistosomiasis according to the Regional Action Plan for Neglected Tropical Diseases in the Western Pacific (20122016), and in 2011 according to the Ministry of Health a decrease of $3.4 \%$ was reported compared to the number of cases in $2010^{12}$. The death estimates due to schistosomiasis need to be re-assessed, as it varies between 24,067 and 200,000 globally per year ${ }^{8}$.

\section{Pathogenesis}

There are five schistosome species that can cause infection in humans, but the ones that affect the intestinal tract are: $S$. mansoni found in Africa and South America, S. japonicum in East Asia, S. mekongi in Laos, Cambodia; and $S$. intercalatum in the West and Central Africa ${ }^{13}$.

The schistosoma japonicum's life cycle starts when the eggs are eliminated with feces, hatch in the water and release the larvae (miracidia) that are viable for up to seven days until they penetrate the snail oncomelania ${ }^{14}$. In the snail there will be two generations of sporocysts and cercariae.

Once released from the snail, the cercariae will swim and penetrate through the skin, shed the tail and become a schistosomulae; they become adult worms and migrate to the mesenteric venules of the small and large intestine $^{14}$. Finally, the circle starts again when the eggs move toward the lumen of the intestine and pass with the feces. This life cycle includes snails, domestic and wild animals which makes its control and prevention more complicated; therefore, treating the infection and reducing the oncomelania snail burden is not enough ${ }^{15}$.

The physiopathology of chronic schistosomiasis results from the eggs lodging, inducing immune response, granuloma formation, fibrotic changes, damaging the organs and tissues of the human host ${ }^{16}$. Adult worms absorb host proteins so if not attacked by the immune system, they can live coated with host antigens for years ${ }^{17}$. The shed eggs penetrate the bowel adjacent to mesenteric vessels, whereas unshed eggs go back to portal circulation, lodge and induce granulomatous reaction ${ }^{16}$. In the liver the chronic progression causes periportal fibrosis, called
Symmers piperstem fibrosis, if left untreated it will lead to portal hypertension with all the cirrhosis complications ${ }^{18}$.

\section{Clinical Manifestations}

Clinical manifestations depend on the burden and stage of the disease, the acute phase occurs when cercariae penetrate the skin and produces dermatitis called the swimmer's itch ${ }^{19}$; the chronic phase could present months to years after primary exposure, some do not have a clear history of acute phase or just mild and unspecific symptoms ${ }^{14}$. After 2-8 weeks of the contact with $s$. japonicum, the migration and maturation of the schistosomulae may produce a systemic inflammatory reaction called Katayama fever, with flu-like symptoms, hepatosplenomegaly, and eosinophilia ${ }^{20}$.

Schistosoma japonicum causes intestinal manifestations with fatigue, abdominal pain, cramping, anorexia, diarrhea, and dysentery ${ }^{21}$. When there is heavy infestation the symptoms could be severe and may lead to intestinal ulceration, bleeding, anemia, intestinal polyps, dysplasia and even bowel strictures ${ }^{14,21,22}$.

If $S$. japonicum remains untreated, periportal fibrosis could lead to portal hypertension and gastrointestinal bleeding ${ }^{23}$. People with hepatitis B or C and chronic hepatic injury due to schistosomiasis have more risk for a rapid progression to liver disease or development of hepatocellular carcinoma ${ }^{24}$.

\section{Diagnosis}

For decades the gold standard for diagnosis of schistosomiasis infection has been the detection of eggs in fecal smear and the concentration techniques with KatoKatz thick smear demonstrate the number of eggs excreted daily ${ }^{25}$. Among people living in endemic areas the approach should be through egg and antigen detection ${ }^{25}$.

The egg viability test observes hatching miracidia and assesses the treatment effectiveness, so an active infection is when viable eggs are observed whereas no viable eggs and absence of miracidia correlates with past infection or effective treatment ${ }^{16}$.

General laboratory examination includes the following and the possible findings are: cell blood count within normal ranges, CBC with anemia or eosinophilia (acute), thrombocytopenia (splenic sequestration), liver function tests could show in the chronic stage a mild elevation of alkaline phosphatase and gamma glutamyl transferase $\mathrm{e}^{26,27}$.

For travelers the approach is not the same, serology is the most sensitive test because the parasite burden is low, and it is more useful than egg or antigen detection ${ }^{28}$. Crude extracts of eggs or adult worms are used as antigen in an ELISA test and antibodies to eggs can be detected from six weeks after exposure ${ }^{29}$ 
Another test is the real-time PCR, in stool samples can be $94 \%$ sensitive and $99.9 \%$ specific and may offer added value in diagnosing imported schistosomiasis ${ }^{30}$. The genusspecific PCR can detect all schistosoma species that are infectious to humans and performs very well with feces and urine, but not in serum ${ }^{31}$.

In chronic hepatoesplenic schistosomiasis due to $s$. japonicum the ultrasonography can report periportal fibrosis around portal vein like a "bull's eye", hypertrophy of the left hepatic lobe, atrophy of the right lobe, granulomas, splenomegaly and other portal hypertension signs ${ }^{32}$. Computed tomography and magnetic resonance imaging can demonstrate heterogeneity of hepatic parenchyma, periportal fibrosis, and the presence of venous collateral pathways ${ }^{33}$.

Biopsy is an useful diagnostic tool in the setting of intestinal schistosomiasis, it is more sensitive than stool microscopy and it can demonstrate granulomas surrounding eggs even when stool samples are negative ${ }^{34}$. At an early stage bowel wall biopsy can report mucosal edema, hemorrhage and ulceration, whereas in advanced stage it can detect a thickened bowel wall, polyps, and even strictures $^{34}$.

\section{Treatment}

The treatment for schistosomiasis has important objectives such as reversing acute or early chronic disease and preventing complications. The treatment in the early chronic stage can reverse periportal fibrosis and portal hypertension, but it cannot reverse late stage fibrosis and cirrhotic complications such as esophageal varices ${ }^{35}$.

Praziquantel is the drug of choice for the treatment of all schistosoma species, it acts against adult schistosoma worms with the purpose to reduce egg production but has poor activity against immature schistosoma larvae ${ }^{36}$. Therefore, the treatment is mostly effective from four to six weeks after exposure when the schistosoma is a mature worm, corroborated in an animal study that shows no pharmacological effect within 21 first days ${ }^{26,37}$.

Praziquantel's mechanism of action is on the tegumentary and muscular tissue, causing contractions in the parasite that are followed by its death and also it inhibits the egg production in female parasites ${ }^{38,39}$. The standard dose is $40 \mathrm{mg} / \mathrm{kg}$, but for s. japonicum the dose can be $60 \mathrm{mg} / \mathrm{kg}^{39}$. A cure rate between 65 to $90 \%$ has been described after one regimen ${ }^{40}$. After treatment, follow up in endemic areas should use stool smear after six weeks of treatment; in nonendemic areas since there is no risk for reinfection the control examination can be delayed three to six months ${ }^{29}$. If there is presence of viable eggs after the treatment, the same dose of initial therapy should be prescribed ${ }^{41}$.
Over decades the drug resistance has been a concern; researchers have identified field and experimental isolates that exhibit significantly reduced susceptibility but still the criteria used to classify a resistant strain or evidence of resistance remains controversial ${ }^{42,43}$.

Schistosoma candidate vaccines are being identified through bioinformatics, OMICs approaches ${ }^{44}$. At least three vaccines (Sm14, Sm-TSP-2 and SM-p80 antigens) in the near future will go through human clinical trials ${ }^{45}$, still an efficacious vaccine has not yet been realized.

\section{Prevention}

Strategies to control schistosomiasis in endemic areas include water sanitation, minimizing contact with fresh water, community health-education, eradication of snail species, domestic animals care and treatment, proper work equipment to people at risk of expositiont ${ }^{11}$. Mass treatment has demonstrated to reduce the prevalence and morbidity of schistosomiasis by keeping the parasite load low and by increasing resistance to reinfection ${ }^{46}$.

With the fast economy and technology development in China, the surveillance systems for prevention and control have improved and a great progress has been made in the last six decades ${ }^{47}$. Several interventions to reduce transmission from domestic animals, snails to humans have been adopted as national strategies in China ${ }^{11,47}$.

\section{Conclusion}

Schistosomiasis is one of the neglected tropical diseases currently affecting mostly the poor population and causing thousands of deaths annually worldwide. Schistosoma japonicum is found in China, Indonesia, Philippines, and its clinical manifestations depend on the acute or chronic phase, but if left untreated irreversible complications occur. The prognosis for the intestinal curation is optimistic after the administration of praziquantel.

Despite the regional governments and the World Health Organization efforts to maintain surveillance and treatment policies, more attention and support should be paid to this endemic disease, ensuring that the affected population not only receives suitable treatment but also the education and preventive measures that will not expose them to suffer this parasitic disease. More support should be done to prevent resistance to praziquantel and develop a secure vaccine accessible to everyone.

We encourage the education to endemic areas' population to seek medical advice when gastrointestinal manifestations occur, to medical physicians to consider intestinal schistosomiasis as a differential diagnose when referring gastrointestinal symptoms. 


\section{References}

1. Sah VK, Wang L, Min X, et al. Human schistosomiasis: a diagnostic imaging focused review of a neglected disease. Radiol Infect Dis. 2015; 2(1): 150-157.

2. Cohen JP, Sturgeon G, Cohen A. Measuring progress in neglected disease drug development. Clin Ther. 2014; 36 (7): 1037-1042.

3. Siqueira L, Ferreira D, Siqueira C. Schistosomiasis: Drugs used and treatment strategies. Acta Tropica. 2017; 176: 179-187.

4. Clerinx J, Soentjens P. Epidemiology, pathogenesis, and clinical manifestations of schistosomiasis Uptodate. 2017.

5. Merrifield M, Hotez P, Beaumier CM, et al. Advancing a vaccine to prevent human schistosomiasis. Vaccine. 2016; 34(26): 2988-2991.

6. Xiang JJ, Cheng BJ, Tian F, et al. Perforation of small bowel caused by Schistosoma japonicum: A case report. World J Gastroenterol. 2015; 21(9): 2862-2864.

7. Mott KE, Desjeux P, Moncayo A, et al. Parasitic diseases and urban development. Bull World Health Organ. 1990; 68(6): 691-8.

8. Global Health Estimates 2015: Deaths by Cause, Age, Sex, by Country and by Region, 2000-2015. http://www.who.int/healthinfo/global burden_disease/estimates/en/index1.html Geneva, World Health Organization; 2019.

9. Colley DG, Bustinduy AL, Secor WE, et al. Human schistosomiasis. Lancet. 2014; 383: 2253-54.

10. Olveda DU, Olveda RM, McManus DP, et al. The chronic enteropathogenic disease schistosomiasis. Int J Infect Dis. 2014; 28 193-203.

11. Balen J, Zhao ZY,Williams GM, et al. Prevalence, intensity and associated morbidity of Schistosoma japonicum infection in the Dongting Lake region, China. Bulletin of the World Health Organization. 2007; 85(7): 519-526.

12. World Health Organization. Schistosomiasis in China. WHO Western Pacific Region; 2015. Available at: http://www.wpro.who.int/ china/mediacentre/factsheets/schistosomiasis/en/. [Accessed 26 December 2018].

13. Rudge JW, Webster JP, Lu DB, et al. Identifying host species driving transmission of schistosomiasis japonica, a multihost parasite system, in China. Proc Natl Acad Sci U S A. 2013; 110(28): 11457-62.

14. Gryseels B, Polman K, Clerinx J, et al. Human schistosomiasis. Lancet. 2006; 368(9541): 1106.

15. Corachan M. Schistosomiasis and International travel. Clin Infect Dis. 2002; 35 (4): 446-450.

16. $\mathrm{Gu} \mathrm{K}, \mathrm{Li} \mathrm{Y}$, Rodriguez $\mathrm{P}$, et al. Clinical diagnostic value of viable Schistosoma japonicum eggs detected in host tissues. BMC Infectious Diseases. 2017; 17: 244

17. Mott KE, Desjeux P, Moncayo A, et al. Parasitic diseases and urban development. Bull World Health Organ. 1990; 68(6): 691-8.

18. Lamyman MJ, Noble DJ, Narang S, et al. Small bowel obstruction secondary to intestinal schistosomiasis. Trans R Soc Trop Med Hyg. 2006; 100(9): 885.

19. Rodríguez P, Hernández P, Martín-Garre S, et al. Unexpected hosts: imaging parasitic diseases. Insights Imaging. 2017; 8: 101-125

20. Ross AG, Vickers D, Olds GR, et al. Katayama syndrome. Lancet Infect Dis. $2007 ; 7(3): 218$

21. Ross AGP, Bartley PB, Sleigh AC, et al. Schistosomiasis. N Eng J Med. 2002; 346: 1212-9.

22. Mu A, Fernandes I, Phillips D. A 57-Year-Old Woman With a Cecal Mass. Clin Infect Dis. 2016; 63(5): 703-5.
23. Abdel-Wahab MF, Esmat G, Farrag A, et al. Grading of hepatic schistosomiasis by the use of ultrasonography. Am J Trop Med Hyg. $1992 ; 46(4): 403-8$.

24. El-Tonsy MM, Hussein HM, Helal Tel S, et al. Schistosoma mansoni infection: is it a risk factor for development of hepatocellular carcinoma. Acta Trop. 2013; 128(3): 542-547.

25. Colley DG, Andros TS, Campbell CH. Schistosomiasis is more prevalent than previously thought: what does it mean for public health goals, policies, strategies, guidelines and intervention programs. Infect Dis Poverty. 2017; 6: 63.

26. Gray DJ, Ross AG, Li YS, et al. Diagnosis and management of schistosomiasis. BMJ. 2011; 342: d2651.

27. Elbaz T, Esmat Gamal. Hepatic and Intestinal Schistosomiasis: Review. Journal of Adv Research. 2013; 4: 445-452.

28. Soentjens P, Clerinx J, Aerssens A, et al. Diagnosing acute schistosomiasis. Clin Infect Dis. 2014; 58(2): 304.

29. Coltart CE, Chew A, Storrar N, et al. Schistosomiasis presenting in travellers: a 15-year observational study at the Hospital for Tropical Diseases, London. Trans R Soc Trop Med Hyg. 2015; 109: 214-220.

30. Lier T, Simonsen GS, Haaheim $\mathrm{H}$, et al. Novel real-time PCR for detection of Schistosoma japonicum in stool. Southeast Asian J Trop Med Public Health. 2006; 37(2): 257-64.

31. Cnops L, Tannich E, Polman K, et al. Schistosoma real-time PCR as diagnostic tool for international travelers and migrants. Trop Med Int Health. 2012; 17(10): 1208-16.

32. Richter J, Hatz C, Campagne G, et al. Ultrasound in schistosomiasis. A Practical Guide to the Standardized Use of Ultrasonography for the Assessment of Schistosomiasis-related Morbidity. World Health Organization, Geneva. 2000.

33. Bezerra AS, D'Ippolito G, Caldana RP, et al. Chronic hepatosplenic schistosomiasis mansoni: magnetic resonance imaging and magnetic resonance angiography findings. J Acta Radiol. 2007; 48(2): 125.

34. Jun Cao, Wen-Jia Liu, Xin-Yun $\mathrm{Xu}$, et al. Endoscopic findings and clinicopathologic characteristics of colonic schistosomiasis: A report of 46 cases. World J Gastroenterol. 2010; 16(6): 723-727.

35. Richter J. The impact of chemotherapy on morbidity due to schistosomiasis. Acta Trop. 2003; 86(2-3): 161.

36. Doenhoff MJ, Cioli D, Utzinger J. Praziquantel: mechanisms of action, resistance and new derivatives for schistosomiasis. Curr Opin Infect Dis. 2008; 21: 65967

37. Utzinger J, Keiser J, Shuhua X, et al. Combination chemotherapy of schistosomiasis in laboratory studies and clinical trials. Antimicrob Agents Chemother. 2003; 47(5): 1487-95.

38. Cioli D, Pica-Mattoccia L, Basso A, et al. Schistosomiasiscontrol: praziquantel forever. Mol Biochem Parasitol. 2014; 195(1): 23-29.

39. Santos FK. Desenvolvimento e caracterização de carreadores lipídicos nanoestruturados contendo praziquantel $81 \mathrm{f}$. Dissertação (mestrado) - Universidade Estadual Paulista, Faculdade de Ciências Farmacêuticas. 2011.

40. Fenwick A, Rollinson D, Southgate V. Implementation of human schistosomiasis control: Challenges and prospects. Adv Parasitol. 2006; 61: 567-622.

41. Olliaro PL, Vaillant MT, Belizario VJ. A multicentre randomized controlled trial of the efficacy and safety of single-dose praziquantel at $40 \mathrm{mg} / \mathrm{kg}$ vs. $60 \mathrm{mg} / \mathrm{kg}$ for treating intestinal schistosomiasis in the Philippines, Mauritania, Tanzania and Brazil. Negl Trop Dis. 2011; 5: e1165.

42. Wang W, Wang L, Liang Y. Susceptibility or resistance of praziquantel in human schistosomiasis: a review. Parasitol Res. 2012; 111: 18711877. 
43. Vale N, Gouveia MJ, Rinaldi G, et al. Praziquantel for Schistosomiasis: Single- Drug Metabolism Revisited, Mode of Action, and Resistance. Antimicrob Agents Chemother. 2017; 61: e02582-16.

44. Wang LD, Chen HG, Guo JG, et al. A strategy to control transmission of Schistosoma japonicum in China. N Engl J Med. 2009; 360(2): 121.

45. Molehin AJ, Rojo JU, Siddiqui SZ, et al. Development of a schistosomiasis vaccine. Expert Rev Vaccines. 2016; 15(5): 619-627.
46. Mutapi F, Maizels R, Fenwick A, et al. Human schistosomiasis in the post mass drug administration era. Lancet Infect Dis. 2017; 17(2): e42.

47. Zhang LJ, Li SZ, Wen LY, et al. The Establishment and Function of Schistosomiasis Surveillance System Towards Elimination in The People's Republic of China. Advances in Parasitology. 2016; 92: 118137. 